\title{
Approach of the characterization of delamination in mural paintings
}

\author{
By Jean-Charles Candoré*, Jean-Luc Bodnar*, Françoise Depasse*, Nicolas Horny*, Vincent \\ Detalle $^{* *}$, Philippe Grossel*.
}

*Laboratoire d'Energétique et d'Optique, UFR Sciences Exactes et Naturelles, BP 1039, 51687 Reims cedex 02

** Laboratoire de recherche des monuments historiques, 29 avenue du Paris, 77420 Champs sur Marne

\section{Abstract}

Delamination and air voids in renders are among the main sources of deterioration of mural paintings. Those decays are particularly dangerous because they are almost non visible, because underlying, but they can induce falls of parts of the artistic composition depending on their nature and the affected surface.

The traditional technique used by the restorers to localize delaminated areas is acoustic sounding. It consist in a systematic slight knocking of the painted surface with one hand, when the other hand stays in contact with the surface. The vibration induced by the knocking is then perceived both by hear and touch and allows an experienced restorer to determine the extent of the decay, on the basis of an empirical evaluation of the propagation of the vibration. This technique which is qualitative is very simple and does not necessitate any specific equipment, but the results are not very precise and their quality depend a lot on the skills of the restorer. For example, the thickness or the depth of an air void, cannot be detected, when they represent valuable information for the restorer to evaluate the real state of decay of the mural painting and as a consequence a key point for the choice of a suitable restoration technique.

Moreover, a contact with the mural painting, more or less long and powerful, is needed. It can be hazardous if the painting is powdering or weakened by scaling (as it was the case for the "Saint Christophe" of the Campana collection of the Louvre museum, before its restoration); or if the render is too thin (it then can collapse if the knocking is too powerful). Finally, when the surfaces painted are wide, this operation can be long and laborious.

New control methods, destructive or not can thus still find their place in the field of the assistance to the restoration of works of art.

The Laboratory of Energetics and Optics of the University of Reims Champagne Ardennes has worked for approximately 20 years, with the development of a particular non destructive testing method: photothermal radiometry. The principle of this method of analysis is relatively simple : It consists in subjecting the sample to be analyzed with a luminous flow whose absorption produces a local rise in the temperature close to the point of luminous impact, then to observe the variations of flux densities of material using an infra-red optical detection chain. The photothermal signal thus obtained depends on the optical and thermophysical properties of analyzed material, it is possible then to characterize it. This is a nondestructive test, without contact, used for the thermophysical analysis of thin material. It was already implemented to detect and characterize various types of localised or extended defects (delaminations, cracks, inclusions,...), in various types of materials. It seems to be able to be used in the field of the restoration of works of art, for example helping with the restoration of mural paintings, in addition to acoustic sounding. As infrared photothermal thermography has been successfully tested to localize and characterize decays such as delamination or cracking in several materials,

In this work, we studied the possibilities to characterize the depth at which the defect is. Initially, we were interested in the characterization of a defect in plastazote located in a fresco carried out according to the technique of primitive Italian. In this study, we show that 9th International Conference on Quantitative InfraRed Thermography several parameters characteristic of the photothermal response collected during the experimental analysis, are correlated with the variable depth of this inclusion. We show then that the direct use of one of these parameters allows a good approximation the depth at which is an unknown inclusion

In the second part of this study, we were interested in the characterization of an inclined defect located in a plaster sample (better known thermophysical properties than those of the preceding sample), using inverse methods. We present the photothermal model used to characterize the depth of the defect. Then, we show the method gives access to a good order of the depth at which the defect is located. 


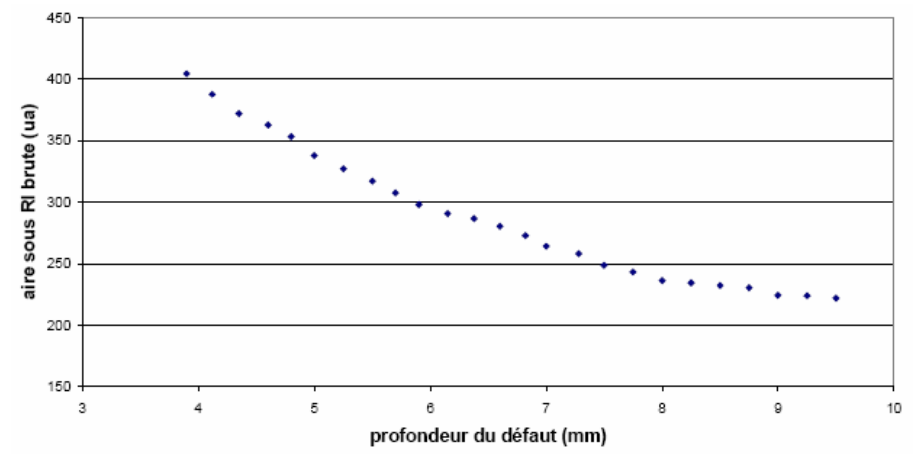

Fig.1. Correlation between the surface contained under the rough impulse responses and the depth at which the defect is. (the sample carried out according to the technique of the primitive Italians)

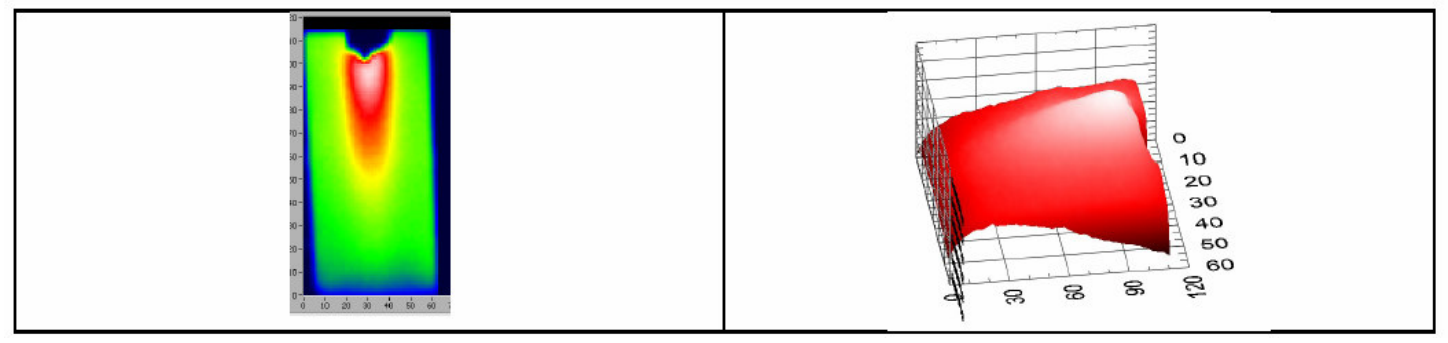

Fig.2. Infra-red images post treated revealing the increasing depth of the defect located in the plaster sample

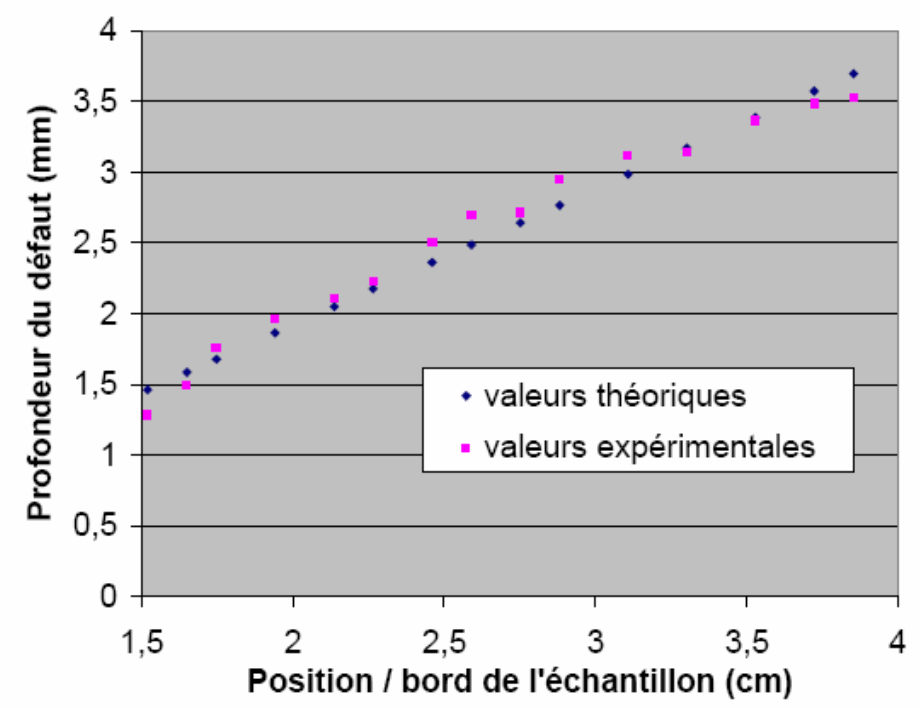

Fig.3. Comparison between the depths of defect measured by inverse method on the plaster sample by stimulated infra-red thermography and using a depth gauge 